\title{
Comparative Analysis of Highly Sensitive PCF for Chemical Sensing in THz Regime
}

\author{
Mohammad Saiful Islam, ${ }^{* 1}$ Anwar Sadath, ${ }^{2}$ Md. Rakibul Islam, ${ }^{3}$ Mohammad Faisal, ${ }^{2}$ \\ ${ }^{1}$ Faculty of Electrical and Electronic Engineering, Bangladesh University of Business and Technology, Dhaka \\ ${ }^{2}$ Faculty of Electrical and Electronic Engineering, Bangladesh University of Engineering and Technology, Dhaka \\ ${ }^{3}$ Faculty of Engineering and Technology, Mymensingh Engineering College, Dhaka
}

Received May 04, 2020; accepted November 09, 2020; published December 17, 2020

\begin{abstract}
Nowadays photonic crystal fiber (PCF) is used for sensing purposes in different fields. In this work, we have proposed a PCF based chemical (Benzene and Ethanol) sensor. Finite Element Method (FEM) based software COMSOL 5.3a is used to investigate the numerical characteristics for the proposed structure. From the numerical analysis, we obtained high sensitivity with low losses for an optimum core diameter of $210 \mu \mathrm{m}$. Our proposed PCF works on a broad range of core diameters and $\mathrm{THz}$ frequency spectra. The fabrication of this model is very simple due to its simplistic design structure.
\end{abstract}

Photonic Crystal Fiber (PCF) was first invented in 1996 and brought about a revolutionary breakthrough in optical fiber technology. PCFs have drawn researcher's attention due to their various favourable features. In telecom and non-telecom applications, PCFs are used at a greater extent as a transmission medium. In recent years, PCFs have been used for several applications like gas sensing, chemical sensing, temperature sensing, salinity sensing and so on. All these applications use light spectrum guiding through the core of PCF. Light pulses travel through the core of PCF based on a total internal reflection (TIR) mechanism. Due to high sensitivity along with their low loss feature and cheapness, PCF sensors are now chosen over electric sensors. Temperature, motion, electric and magnetic field, torsion and pressure can be detected using PCFs. Moreover, flammable gas/chemical leakage detection is a very important safety issue for the chemical/gas production industry. Researchers are now paying more attention to the PCF based sensors due to their robustness combined with high sensitivity. In the past, most of the PCFs were silica glass based but nowadays several materials (Topas, Zeonex, ZBLAN) are being at a greater extent due to their excellent characteristics. These PCFs operate in the $\mathrm{THz}$ frequency spectrum which lies between the microwave and infrared frequency region and cannot be detected by the naked eye. $\mathrm{THz}$ radiation is not detrimental to a human body due to its low photon energy and used in medical imaging and sensing purposes. In addition, $\mathrm{THz}$ radiation can be used to find out any explosives in a highly secured area. In this work, we have proposed a hexagonal shaped PCF with 3 ellipses inside the core in order to achieve high relative sensitivity, low confinement loss and less effective material loss. We have targeted to detect two chemicals $($ Benzene $=1.366$, Ethanol $=1.354$ ) using our proposed model because ethanol causes headaches, confusion, dizziness and nausea. On the other hand, benzene causes a decrease in red blood cells, excessive bleeding and immune system disorder etc. Zeonex is selected as the base material and 3 ellipses are introduced as the chemical to be sensed. The pitch (hole to hole distance) of the proposed fiber is defined as $p$ and $r$ is defined as the radius of each circle used as an air hole in the cladding region. We have selected the pitch value as $p=2.1 r$ to avoid the overlapping of air holes and maintain symmetry in the cladding region to avoid any fabrication difficulty.

An artificial boundary condition named as perfectly matched layer (PML) is attached outside of the cladding region to truncate the computational domain of the proposed fiber. Figure 1. shows the cross-sectional view of the proposed chemical sensor.

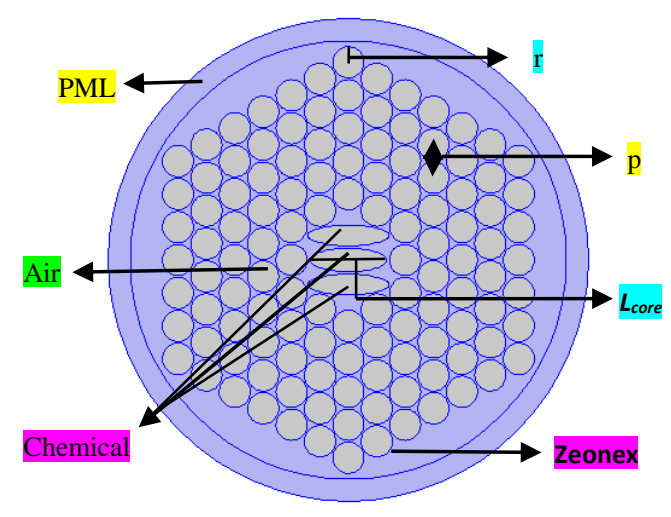

Fig. 1. Cross-sectional view of the proposed chemical sensor.

*E-mail: saiful.eee14@gmail.com 


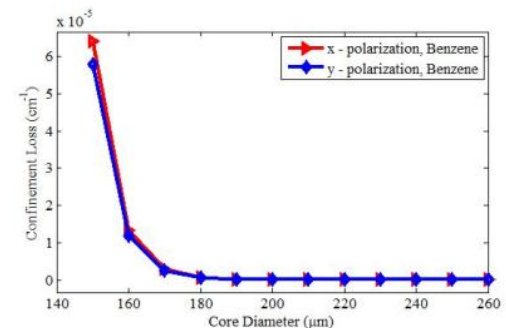

Fig. 2. Confinement loss vs Core Diameter.

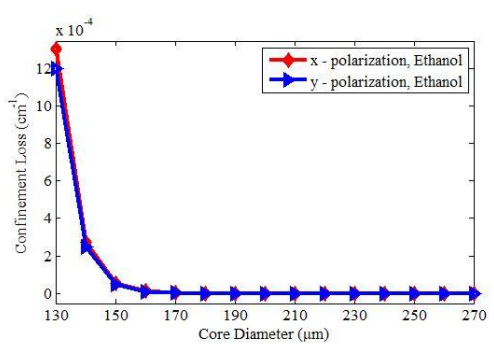

Fig. 5. Confinement loss vs Core Diameter.

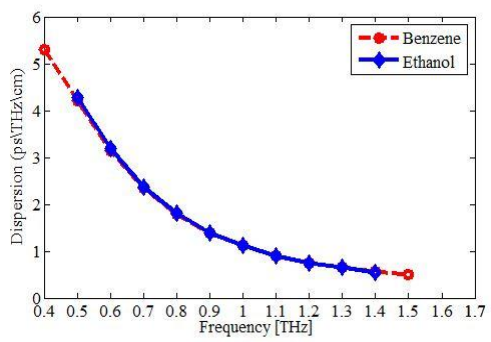

Fig. 8. Dispersion vs Frequency.

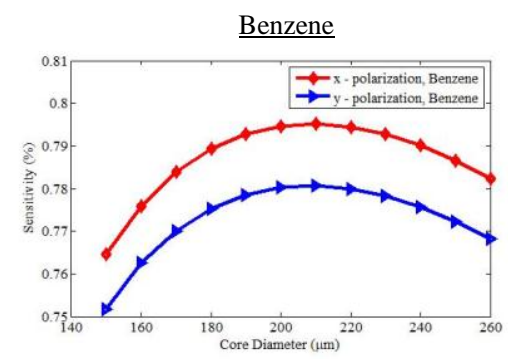

Fig. 3. Sensitivity vs Core Diameter.

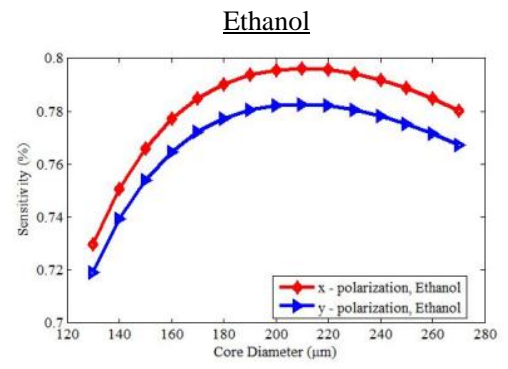

Fig. 6. Sensitivity vs Core Diameter.

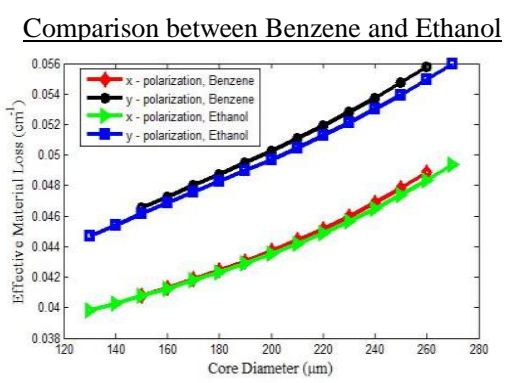

Fig. 9. EML vs Core Diameter.

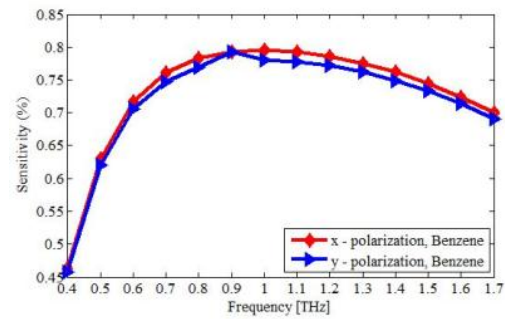

Fig. 4. Sensitivity vs Frequency.

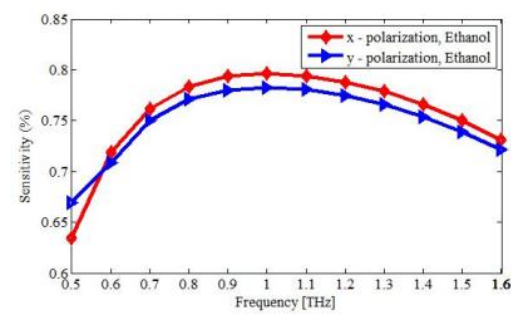

Fig. 7. Sensitivity vs Frequency.

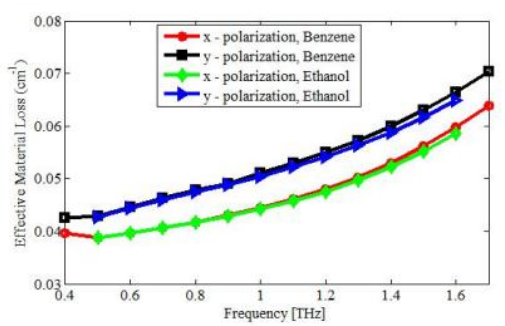

Fig. 10. EML vs Frequency.
We have chosen Zeonex as the background material because of its constant refractive index $\left(n_{\text {mat }}=1.53\right)$ and low absorption loss. To obtain high accuracy of numerical analysis, we solved Maxwell's equations selecting the fine mesh. To assure perfect propagation by the TIR mechanism, we have selected the refractive index of 1.366 for benzene and 1.354 for ethanol respectively. Comparison between two PCFs is also shown for sensing applications by Ademgil (2014). The leakage light from the core region to the exterior materials in PCFs defined as confinement loss can be found from the imaginary part of $n_{\text {eff }}$ using the following equation [1]:

$$
L_{c}=\frac{4 \pi f}{\mathrm{c}} \times \operatorname{Im}\left(n_{e f f}\right), \mathrm{cm}^{-1}
$$

where $f, \operatorname{Im}\left(n_{\text {eff }}\right)$ and $c$ represent the frequency, imaginary part of the refractive index and velocity of the light in free space. Confinement loss can strongly be affected by the diameter of the air holes used in the cladding (Olyaee and Naraghi 2013). The interaction of light with material to be sensed, known as the effective sensitivity can be calculated using the following equation [2]:

$$
r=\frac{n_{r}}{n_{e f f}} \times f
$$

Here, $n_{r}, n_{\text {eff }}$ represent the material refractive index that needs to be sensed and modal effective index respectively. In addition, $f$ represents the percentage ratio of air hole power to total power which can be obtained by the following equation [3]:

$$
f=\frac{f_{\text {sample }} \operatorname{Re}\left(E_{x} H_{y}-E_{y} H_{x}\right) d x d y}{f_{\text {total }} \operatorname{Re}\left(E_{x} H_{y}-E_{y} H_{x}\right) d x d y} \times 100 \%
$$

Here, both transverse electric field and magnetic field are defined by $E_{x} H_{y}$ and $E_{y} H_{x}$, respectively.

Though most of the materials are highly absorbent to the $\mathrm{THz}$ frequency, it is very important to figure out the effective material loss for the fiber which can be measured by the following equation [5]: 


$$
\alpha_{e f f}=\sqrt{\frac{\varepsilon_{0}}{\mu_{0}}}\left(\frac{\int_{m a t} n_{m a t}|E|^{2} \alpha_{m a t} d A}{\left|\int_{a l l} S_{z} d A\right|}, \mathrm{cm}^{-1}\right.
$$

where, $\varepsilon_{0}$ and $\mu_{0}$ are the relative permittivity and permeability respectively, $\alpha_{\text {mat }}$ is the material loss, $S_{z}$ is the pointing vector of the $z$ component, $n_{\text {mat }}$ is the refractive index of the background material and $\mathrm{E}$ denotes the electric field, respectively. Another important parameter of PCFs is dispersion which measures the amount of change between the original and distorted version of a signal and can be found from the following equation [5]:

$$
\beta_{2}=\frac{2}{c} \frac{d_{n_{e f f}}}{d \omega}+\frac{\omega}{c} \frac{d^{2} n_{e f f}}{d \omega^{2}}, p s / T H z / c m
$$

Here, $\mathrm{c}$ is the velocity of light and $\omega=2 \pi f$. We have obtained nearly zero confinement loss for both chemicals which are presented in Fig. 2 and Fig. 5. obtained nearly flat dispersion of $1.114 \pm 0.3023$ $\mathrm{ps} / \mathrm{THz} / \mathrm{cm}$ for benzene and $1.035 \pm 0.2811 \mathrm{ps} / \mathrm{THz} / \mathrm{cm}$ for ethanol, respectively, for our proposed PCF. This is clearly shown in Fig. 8. It is experimentally found that effective material loss depends on the frequency according to the Empirical formula [4]: $\alpha(v)=v 2+0: 63 v$ $0: 13[\mathrm{~dB} / \mathrm{cm}$ ]. From Fig. 9 and Fig. 10, it is evident that the effective material loss increases with the increase in both core diameter and frequency. We have found the value of effective material loss of $0.04441 \mathrm{~cm}^{-1}$ ( $x$ polarization mode) and $0.05107 \mathrm{~cm}^{-1}$ ( $y$-polarization mode) for benzene at optimum core diameter and frequency. Furthermore, $0.04419 \mathrm{~cm}^{-1}$ ( $x$-polarization mode) and $0.05047 \mathrm{~cm}^{-1} \quad(y$-polarization mode), respectively, are found for ethanol at the same optimum core diameter and frequency.

Various fabrication processes such as 3D printing technology, sol-gel technique, stack and draw, capillary stacking, drilling and extrusion method allow to fabricate

Table 1. Comparison of the proposed sensor with prior PCF sensors

\begin{tabular}{|c|c|c|c|c|}
\hline Ref. & $\begin{array}{c}\text { Relative Sensitivity } \\
(\%)\end{array}$ & $\begin{array}{c}\text { Confinement loss } \\
\left(\mathrm{cm}^{-1}\right)\end{array}$ & $\begin{array}{c}\alpha e f f \\
\left(\mathrm{~cm}^{-1}\right)\end{array}$ & Background Material \\
\hline$[1]$ & 53.35 & $1.62 \times 10^{-10}$ & - & Silica \\
\hline$[2]$ & - & $5.42 \times 10^{-13}$ & 0.066 & Topas \\
\hline$[3]$ & 49.17 & $2.75 \times 10^{-10}$ & - & Silica \\
\hline Proposed & 79 & $6.87 \times 10^{-9}$ & 0.04441 & Zeonex \\
\hline
\end{tabular}

By increasing the number of air holes in the cladding region, the confinement loss can be greatly reduced [2]. We can see from Fig. 2 and Fig. 5 that after a specific core diameter $(170 \mu \mathrm{m} \div 180 \mu \mathrm{m})$, the confinement loss becomes constant. For Benzene, we have obtained a very low confinement loss of $6.874 \times 10^{-09} \mathrm{~cm}^{-1}$ and $5.617 \times$ $10^{-09} \mathrm{~cm}^{-1}$ for $x$ and $y$ polarization mode respectively at optimum core diameter. On the other hand, for Ethanol it is about $6.574 \times 10^{-09} \mathrm{~cm}^{-1}$ and $5.537 \times 10^{-09} \mathrm{~cm}^{-1}$ for $x$ and $y$ polarization respectively. The sensitivity of both chemicals (Benzene and Ethanol) increases with the increase of core diameter which is depicted in Fig. 3 and Fig. 6, respectively. After a certain point, the sensitivity of the proposed model decreases. Moreover, from both Fig. 4 and Fig. 7, we find that sensitivity also increases with the increase in frequency. However, after $L_{\text {core }}=210 \mu \mathrm{m}$ (core length) and $1.0 \mathrm{THz}$ frequency, the sensitivity starts waning. This is the reason why we have considered $210 \mu \mathrm{m}$ as optimum core and $1.0 \mathrm{THz}$ as optimum operating frequency. For the optimum core length at $1.0 \mathrm{THz}$ frequency, we have found $79 \%$ sensitivity for $\mathrm{x}$-polarization mode and $78 \%$ sensitivity for $y$-polarization mode for both chemicals. From the numerical investigation of our proposed fiber, we have any sort of PCFs [1]. Using 3D printing technology and extrusion method, it is possible to fabricate almost all complex forms.

In our work, we have proposed a Zeonex based PCF sensor to detect Benzene and Ethanol. We have obtained a very high sensitivity of $79 \%$ for $x$-polarization and $78 \%$ for $y$-polarization mode for both chemicals with a nominal confinement loss and tolerable dispersion variation. Easy fabrication due to its simple structure and low loss features make our proposed PCF a potential candidate for sensing applications in the $\mathrm{THz}$ frequency spectrum.

\section{References}

[1] Md.F.H. Arif, Md.J.H. Biddut, Sensing Bio-Sensing Res. 12, 8 (2017).

[2] P. Kumar, Md.H. Bikash, K. Ahmed, S. Sen, Photonics 6(1), 32 (2019).

[3] S. Asaduzzaman, K. Ahmed, T. Bhuiyan, T. Farah, SpringerPlus 5, 748 (2016).

[4] Md.S. Islam, J. Sultana, J. Atai, D. Abbott, S. Rana, M.R. Islam, App. Opt. 56(4), 1232 (2017).

[5] S. Atakaramians, S. Afshar, H. Ebendorff-Heidepriem, M. Nagel, B.M. Fischer, D. Abbott, T.M. Monro, Opt. Expr. 17(16), 14053 (2009). 